\title{
SOME OBSERVATIONS ON BROKEN SYMMETRIES
}

\author{
M. Testa ${ }^{1,2}$ \\ 1 Theory Division, CERN, 1211 Geneva 23, Switzerland*. \\ ${ }^{2}$ Dipartimento di Fisica, Università di Roma "La Sapienza" \\ Sezione INFN di Roma \\ P.le A. Moro 2, 00185 Roma, Italy ${ }^{\star \star}$.
}

\begin{abstract}
We present a general analysis of the field theoretical properties which guarantee the recovery, at the renormalized level, of symmetries broken by regularization. We also discuss the anomalous case.
\end{abstract}

* Address until August 31st, 1998.

$\star \star$ Permanent address. 


\section{Introduction}

The situation in which a classical global symmetry is broken through regularization is far from exceptional in field theory and is relevant for basic symmetries such as chirality and scale invariance. In these cases either the symmetry is restored in the infinite cutoff renormalized theory or quantum anomalies definitively spoil the conservation laws of the classical action.

While for global symmetries respected by the regulator the bare Noether currents are finite and correctly normalized, the situation is different for classical symmetries violated by the regularization. In this case, in fact, even if the symmetry will be recovered in the renormalized theory, the corresponding currents require finite renormalization. In the presence of anomalies, instead, infinite, scale dependent renormalizations are needed in order to define the corresponding currents.

The general requisites underlying the recovery of the non-anomalous $S U(3) \otimes S U(3)$ chiral symmetry and the corresponding classification of the local observables, have been discussed in ref. [1] in the context of the lattice discretization with Wilson fermions]. In the case of octet chiral currents, some of these requirements, those underlying the construction of the conserved currents, have been shown[2] to be satisfied at any order in perturbation theory. It must be said, however, that checks within perturbation theory, although important, do not provide an "explanation" why these prerequisites are indeed satisfied: one is left with the impression that the theory is, for some mysterious reason, ready to incorporate the symmetry, although broken for any finite cutoff.

It is the purpose of this paper to explore the mechanisms underlying the recovery of a symmetry, without invoking perturbation theory, but only using general properties expected to be non-perturbatively valid in any field theory. In other words, we will try to trace back to some general field theoretical features the various properties which, in ref. [1], were conjectured from the analysis of one loop perturbation theory.

Let me state explicitly the general euclidean field theoretical assumptions which I will use (at least those of which I am aware):

1. the renormalization structure is the same as the one found in perturbation theory;

2. renormalized Green's function are finite when computed at non-exceptional external momenta and obey the Callan-Symanzik equation[3]; ;

3. the theory exists in the zero mass limit, in the sense that Green's functions of "suitably renormalized operators" 2 are finite when computed at non-exceptional momenta沟;

4. the regularized Green's functions are finite at non-exceptional momenta, also in the massless limit .

\footnotetext{
${ }^{1}$ As repeatedly stressed, the discussion presented in ref. [1] is not restricted to lattice regularization, but is indeed quite general and it is relevant whenever the process of regularization breaks a symmetry of the classical action.

${ }^{2}$ This means that the renormalization conditions should not introduce spurious, mass-dependent i.r. divergent terms like, e.g. $\log \left(p^{2} / m_{q}^{2}\right)$. The i.r. safe renormalization conditions are the usual ones performed at a scale $\mu$.
} 
The above properties are known to be true to all orders in perturbation theory. If they will ever turn out to have a non-perturbative validity, the same will be true for the considerations exposed in the following sections. Assumption 4 is, however, on a different ground: it concerns the regularization. Although valid for any decent regularization, we will show that it is not necessary in the proof of the recovery of the symmetry, even in the massless limit: assumption 1 will only be used to exclude some pathological behaviour, not found in lattice perturbation theory, of the renormalization constants of any operator.

The plan of the paper is as follows. In Section 2 we give a simple non-perturbative derivation of a fundamental, well-known, order by order in perturbation theory, property concerning composite operators: power divergent mixings with lower dimensional operators never depend on the renormalization scale $\mu$. Starting from this result we will discuss, in Section 3, the case of Ward Identities related to global non-anomalous symmetries, showing their validity in the renormalized theory. Finally, in Section 1 , we will discuss the case of the chiral $U(1)$ global anomalous symmetry and its Ward Identities.

\section{Power Divergent Operators}

We start considering a theory with fundamental fields $\phi(x)$, regularized by a cutoff] $\Lambda=1 / a$, defined by a certain number of (bare) coupling constants which we collectively denote by $g_{0}$. The theory is renormalized at a subtraction point $\mu$ and the renormalized Green's functions, $G^{(n)}(x ; g, \mu)$, are expressed in terms of a set of renormalized couplings $g$ :

$$
G^{(n)}(x ; g, \mu) \equiv \frac{1}{\left[Z_{\phi}\left(g_{0}, a \mu\right)\right]^{n / 2}}\left\langle\phi\left(x_{1}\right) \ldots \phi\left(x_{n}\right)\right\rangle \equiv\left\langle\phi^{(r e n)}\left(x_{1}\right) \ldots \phi^{(r e n)}\left(x_{n}\right)\right\rangle
$$

where $Z_{\phi}\left(g_{0}, a \mu\right)$ is the wave function renormalization of $\phi(x), \phi^{(r e n)}(x)$ denote the renormalized fields and $\langle\ldots\rangle$ is the euclidean expectation value with respect to the regularized measure.

Just to establish some notation, let us recall that the Callan-Symanzik[3] differential operator, $\left.\mu \frac{d}{d \mu}\right|_{g_{0}, a}$ acting on $G^{(n)}(x ; g, \mu)$ gives:

$$
\begin{aligned}
& \left.\mu \frac{d}{d \mu}\right|_{g_{0}, a} G^{(n)}(x ; g, \mu) \equiv\left(\mu \frac{\partial}{\partial \mu}+\beta(g) \frac{\partial}{\partial g}\right) G^{(n)}(x ; g, \mu)= \\
& =\left[\left.\mu \frac{d}{d \mu}\right|_{g_{0}, a} \frac{1}{\left[Z_{\phi}\left(g_{0}, a \mu\right)\right]^{n / 2}}\right]\left\langle\phi\left(x_{1}\right) \ldots \phi\left(x_{n}\right)\right\rangle= \\
& =-n \gamma_{\phi}(g) G^{(n)}(x ; g, \mu)
\end{aligned}
$$

where, as usual, we denoted by $\left.\gamma_{\phi}(g) \equiv \frac{1}{2} \mu \frac{d}{d \mu}\right|_{g_{0}, a} \log \left[Z_{\phi}\left(g_{0}, a \mu\right)\right]$ the anomalous dimension of $\phi$. In eq.(2) we exploited the fact that $\left.\mu \frac{d}{d \mu}\right|_{g_{0}, a}$ gives 0 , when acting on bare quantities.

\footnotetext{
${ }^{3}$ We will, for definiteness, adopt the language of lattice regularization, but, as already stressed, our discussion is completely general.
} 
Let us now consider the case of a composite operator $O(x)$. In order to make it finite it has to be mixed with bare operators of equal or smaller dimension. In order to simplify the presentation of the argument we consider a simple situation in which only mixings with lower dimensional operators occur.

In this case we have:

$$
O_{R}(x)=Z_{O}\left[O(x)+\frac{\tilde{Z}}{a} \tilde{O}(x)\right]
$$

where we schematically denoted by $\tilde{O}(x)$ the lower dimensional operators.

The dimensionless coefficients $Z_{O}$ and $\tilde{Z}$ are chosen, according to appropriate renormalization conditions at the scale $\mu$, so that the Green's functions:

$$
G^{\left(O_{R}, n\right)}\left(x, x_{1} \ldots x_{n}\right) \equiv \frac{1}{\left[Z_{\phi}\left(g_{0}, a \mu\right)\right]^{n / 2}}\left\langle O_{R}(x) \phi\left(x_{1}\right) \ldots \phi\left(x_{n}\right)\right\rangle
$$

stay finite, together with their Fourier transforms, as $a \rightarrow 0$.

Applying $\left.\mu \frac{d}{d \mu}\right|_{g_{0}, a}$ to both sides of eq.(44), we get:

$$
\begin{aligned}
& \left.\mu \frac{d}{d \mu}\right|_{g_{0}, a} G^{\left(O_{R}, n\right)}\left(x, x_{1} \ldots x_{n}\right) \equiv \\
& \equiv\left(\mu \frac{\partial}{\partial \mu}+\beta(g) \frac{\partial}{\partial g}\right) G^{\left(O_{R}, n\right)}\left(x, x_{1} \ldots x_{n}\right)= \\
& =-n \gamma_{\phi}(g) G^{\left(O_{R}, n\right)}\left(x, x_{1} \ldots x_{n}\right)+ \\
& +\frac{\left[\left.\mu \frac{d}{d \mu}\right|_{g_{0}, a} Z_{O}\right]}{Z_{O}} G^{\left(O_{R}, n\right)}\left(x, x_{1} \ldots x_{n}\right)+ \\
& +\frac{Z_{O}}{a}\left[\left.\mu \frac{d}{d \mu}\right|_{g_{0}, a} \tilde{Z}\right]\left\langle\tilde{O}(x) \phi_{R}\left(x_{1}\right) \ldots \phi_{R}\left(x_{n}\right)\right\rangle \equiv \\
& \equiv-\left(n \gamma_{\phi}(g)+\gamma_{O}(g)\right) G^{\left(O_{R}, n\right)}\left(x, x_{1} \ldots x_{n}\right)+ \\
& +\frac{Z_{O}}{a}\left[\left.\mu \frac{d}{d \mu}\right|_{g_{0}, a} \tilde{Z}\right]\left\langle\tilde{O}(x) \phi_{R}\left(x_{1}\right) \ldots \phi_{R}\left(x_{n}\right)\right\rangle
\end{aligned}
$$

where $\gamma_{O}(g)$ denotes the anomalous dimension of $O_{R}(x)$.

Since the l.h.s. and the first term of the r.h.s. of eq.(5) are finite, we must have:

$$
\left.\mu \frac{d}{d \mu}\right|_{g_{0}, a} \tilde{Z}=0
$$

Eq.(6) proves in complete generality that power divergent subtractions of composite operators do not contribute to anomalous dimensions and that, in presence of dimensionless couplings only, they do not contain any logs.

\section{Global Non-Anomalous Symmetries}

In this section we will deal, for definiteness, with non singlet chiral transformations, softly broken by a mass term $M_{0}$.

\footnotetext{
${ }^{4}$ For simplicity we will consider in the following the flavor-symmetric case $M_{0} \propto I$
} 


\subsection{Ward Identities with Elementary Operator Insertions}

As discussed in ref. [1], the naive chiral variation of the regularized functional integral gives rise to the regularized Ward Identity?:

$$
\begin{aligned}
& \partial_{x}^{\mu}\left\langle A_{\mu}^{a}(x) q(y) \bar{q}(z)\right\rangle=M_{0}\left\langle\bar{q}(x) \lambda^{a} \gamma_{5} q(x) q(y) \bar{q}(z)\right\rangle+ \\
& +\delta^{(4)}(x-y)\left\langle\gamma_{5} \frac{\lambda^{a}}{2} q(y) \bar{q}(z)\right\rangle+\delta^{(4)}(x-z)\left\langle q(y) \bar{q}(z) \frac{\lambda^{a}}{2} \gamma_{5}\right\rangle+ \\
& +\left\langle X^{a}(x) q(y) \bar{q}(z)\right\rangle
\end{aligned}
$$

where

$$
A_{\mu}^{a}(x) \equiv \bar{q}(x) \frac{\lambda^{a}}{2} \gamma_{\mu} \gamma_{5} q(x)
$$

and $X^{a}(x)$ is the chiral variation of the Wilson term. Since $X^{a}(x)$ vanishes in the formal classical limit, it has the form:

$$
X^{a}(x)=a O_{5}^{a}(x)
$$

Eq.(1) has still to be renormalized. In particular the composite operator $O_{5}^{a}(x)$, defined in eq.(9), is not finite as $a \rightarrow 0$, but contains power divergences which can

compensate the overall factor $a$. In order to construct a finite operator, $\bar{O}_{5}^{a}(x)$, out of $O_{5}^{a}(x)$, we must consider appropriate linear combinations, as generically described in eq.(3). In the case of $O_{5}^{a}(x)$, dimension 5 operators will appear with logarithmically divergent coefficients, while lower dimensional operators will contribute with coefficients proportional to inverse powers of the lattice spacing $a$. In order to simplify the presentation we will not write down explicitly the logarithmically divergent mixings. We, therefore, have:

$$
\bar{O}_{5}^{a}(x)=Z_{5}\left\{O_{5}^{a}(x)+\frac{\bar{M}}{a} \bar{q}(x) \lambda^{a} \gamma_{5} q(x)+\frac{\left(Z_{A}-1\right)}{a} \partial^{\mu} A_{\mu}^{a}(x)\right\}
$$

While $Z_{5}\left(g_{0}, a \mu\right)$ is logarithmically divergent, $Z_{A}$ and $\bar{M}$ are restricted by eq.(6), to be of the forml:

$$
\begin{aligned}
& Z_{A}=Z_{A}\left(g_{0}, a M_{0}\right) \\
& \bar{M}=\frac{w\left(g_{0}, a M_{0}\right)}{a}
\end{aligned}
$$

Eq.(7) then becomes [1]:

$$
\begin{aligned}
& \partial_{x}^{\mu}\left\langle\hat{A}_{\mu}^{a}(x) q(y) \bar{q}(z)\right\rangle=m_{q}\left\langle\bar{q}(x) \lambda^{a} \gamma_{5} q(x) q(y) \bar{q}(z)\right\rangle+ \\
& +\delta^{(4)}(x-y)\left\langle\gamma_{5} \frac{\lambda^{a}}{2} q(y) \bar{q}(z)\right\rangle+\delta^{(4)}(x-z)\left\langle q(y) \bar{q}(z) \frac{\lambda^{a}}{2} \gamma_{5}\right\rangle+ \\
& +\left\langle\bar{X}^{a}(x) q(y) \bar{q}(z)\right\rangle
\end{aligned}
$$

\footnotetext{
${ }^{5}$ We adopt the conventions: $\left\{\lambda^{a}, \lambda^{b}\right\}=2 d^{a b c} \lambda^{c}, d^{a b 0}=\delta^{a b}, \lambda^{0}=\frac{2}{3} I$.

${ }^{6}$ Strictly speaking $Z_{5}$ could also depend on $a M_{0}$, if we do not use assumption 4 . See the further discussions on this point, later in this section.

${ }^{7}$ In this case also $M_{0}$ has to be included among the bare couplings.
} 
where:

$$
\begin{aligned}
& \hat{A}_{\mu}^{a}(x) \equiv Z_{A} A_{\mu}^{a}(x) \\
& m_{q} \equiv M_{0}-\bar{M} \\
& \bar{X}^{a}(x) \equiv \frac{a}{Z_{5}} \bar{O}_{5}^{a}(x)
\end{aligned}
$$

The $a M_{0}$ dependence in eqs.(11) and (12) is not further restricted by the renormalization group and looks somewhat problematic, at least for considerations pertaining the massless limit of QCD, reached when:

$$
M_{0}=M_{c r} \equiv \frac{f_{c r}\left(g_{0}\right)}{a} \Leftrightarrow m_{q}=0
$$

In fact eq.(11), does not forbid the presence, in $Z_{A}$, of terms such as $\log \left(a m_{q}\right)$; in ref. [1] the absence of such terms was explicitly assumed and this assumption has afterwards been confirmed in perturbation theory [2]. In our case these terms are excluded by assumption 1 stated in the introduction, which guarantees the existence, in the massless limit, of any regularized bare operator. However, as we will show at the end of this section, this hypothesis does not seem necessary in order to recover the symmetry, even in the massless limit. For the moment, therefore, we will proceed without invoking it.

Using eq.(13) we can now establish, in full generality, the recovery of chiral symmetry in the continuum limit. First of all, since the quark fields appear homogeneously in eq.(13), we can proceed to their renormalization:

$$
\begin{aligned}
& \partial_{x}^{\mu}\left\langle\hat{A}_{\mu}^{a}(x) q^{(r e n)}(y) \bar{q}^{(r e n)}(z\rangle=m_{q}\left\langle\bar{q}(x) \lambda^{a} \gamma_{5} q(x) q^{(r e n)}(y) \bar{q}^{(r e n)}(z)\right\rangle+\right. \\
& +\delta^{(4)}(x-y)\left\langle\gamma_{5} \frac{\lambda^{a}}{2} q^{(r e n)}(y) \bar{q}^{(r e n)}(z)\right\rangle+\delta^{(4)}(x-z)\left\langle q^{(r e n)}(y) \bar{q}^{(r e n)}(z) \frac{\lambda^{a}}{2} \gamma_{5}\right\rangle+ \\
& +\left\langle\bar{X}^{a}(x) q^{(r e n)}(y) \bar{q}^{(r e n)}(z)\right\rangle
\end{aligned}
$$

where:

$$
\begin{aligned}
q^{(r e n)}(x) & \equiv \frac{q(x)}{\sqrt{Z_{q}\left(g_{0}, a \mu\right)}} \\
\bar{q}^{(r e n)}(x) & \equiv \frac{\bar{q}(x)}{\sqrt{Z_{q}\left(g_{0}, a \mu\right)}}
\end{aligned}
$$

In eq.(17) we can safely drop the insertion of $\bar{X}^{a}(x)$, since $\bar{O}_{5}^{a}(x)$ is finite when inserted together with renormalized fundamental fields and $Z_{5}$ behaves, at most, logarithmically. We then get:

$$
\begin{aligned}
& \partial_{x}^{\mu}\left\langle\hat{A}_{\mu}^{a}(x) q^{(r e n)}(y) \bar{q}^{(r e n)}(z)\right\rangle=m_{q}\left\langle\bar{q}(x) \lambda^{a} \gamma_{5} q(x) q^{(r e n)}(y) \bar{q}^{(r e n)}(z)\right\rangle+ \\
& +\delta^{(4)}(x-y)\left\langle\gamma_{5} \frac{\lambda^{a}}{2} q^{(r e n)}(y) \bar{q}^{(r e n)}(z)\right\rangle+\delta^{(4)}(x-z)\left\langle q^{(r e n)}(y) \bar{q}^{(r e n)}(z) \frac{\lambda^{a}}{2} \gamma_{5}\right\rangle
\end{aligned}
$$

Eq.(19) implies, by a standard argument, the separate u.v. finiteness of both $m_{q} \bar{q}(x) \lambda^{a} \gamma_{5} q(x)$ and $\hat{A}_{\mu}^{a}(x)$. In fact, integrating eq.(19) over $x$, we get:

$$
\begin{aligned}
& m_{q} \int d^{4} x\left\langle\bar{q}(x) \lambda^{a} \gamma_{5} q(x) q^{(r e n)}(y) \bar{q}^{(r e n)}(z)\right\rangle= \\
& =-\left[\left\langle\gamma_{5} \frac{\lambda^{a}}{2} q^{(r e n)}(y) \bar{q}^{(r e n)}(z)\right\rangle+\left\langle q^{(r e n)}(y) \bar{q}^{(r e n)}(z) \frac{\lambda^{a}}{2} \gamma_{5}\right\rangle\right]
\end{aligned}
$$


Eq.(20) shows that, in virtue of the (assumed) u.v. finiteness of the Green's functions of renormalized fields, the integrated mass insertion is finite. Since there are no operators of dimension $\leq 3$ with identically vanishing $x$ integral, which could mix with $\bar{q}(x) \lambda^{a} \gamma_{5} q(x)$, we conclude that the non integrated mass insertion in eq.(19) is also finite. This, in turn, shows that $\partial_{x}^{\mu}\left\langle\hat{A}_{\mu}^{a}(x) q^{(r e n)}(y) \bar{q}^{r e n}(z)\right\rangle$ is finite by itself. Since the symmetry is ungauged, there are no operators of dimension $\leq 3$ with identically vanishing 4-divergence which could mix with $\hat{A}_{\mu}^{a}(x)$. This shows that $\hat{A}_{\mu}^{a}(x)$ is finite and correctly normalized, since it satisfies the continuum Ward Identity, eq.(19)[四].

Let me finally discuss the question of the massless limit. I want to show that assumptions 12, alone, imply the existence of $\hat{A}_{\mu}^{a}(x)$ also in the limit $m_{q} \rightarrow 0$. The finiteness of insertions of "suitably renormalized operators" at non-exceptional momenta, assumption 2, cannot be directly invoked here, because the normalization of $\hat{A}_{\mu}^{a}(x)$ has not been chosen "suitably", but has been fixed by the theory itself, through eq.(14). However $\hat{A}_{\mu}^{a}(x)$ must be proportional to a "suitably renormalized operator", possibly through a factor which diverges logarithmically as $m_{q} \rightarrow 0$. This means that, if we can show the finiteness of one particular insertion of $\hat{A}_{\mu}^{a}(x)$ in the massless limit at non-exceptional momenta, then the current itself will be well defined. The argument proceeds as follows. Eq.(20), shows that $m_{q} \int d^{4} x \bar{q}(x) \lambda^{a} \gamma_{5} q(x)$ and its limit for $m_{q} \rightarrow 0$ can never be infinite, because of the assumed finiteness of the Green's functions of the renormalized quark fields appearing in the r.h.s. 8 This implies that, when inserted at non-zero momentum, $m_{q} \bar{q}(x) \lambda^{a} \gamma_{5} q(x)$ has to vanish, as $m_{q} \rightarrow 0$. If we now take the Fourier transform of eq.(19), with respect to $x$, at some momentum $k \neq 0$, for $m_{q} \rightarrow 0$ :

$$
\begin{aligned}
& \int d^{4} x e^{-i k x} \partial_{x}^{\mu}\left\langle\hat{A}_{\mu}^{a}(x) q^{(r e n)}(y) \bar{q}^{(r e n)}(z)\right\rangle= \\
& =i k_{\mu} \int d^{4} x e^{-i k x}\left\langle\hat{A}_{\mu}^{a}(x) q^{(r e n)}(y) \bar{q}^{(r e n)}(z)\right\rangle= \\
& =e^{-i k y}\left\langle\gamma_{5} \frac{\lambda^{a}}{2} q^{(r e n)}(y) \bar{q}^{(r e n)}(z)\right\rangle+e^{-i k z}\left\langle q^{(r e n)}(y) \bar{q}^{(r e n)}(z) \frac{\lambda^{a}}{2} \gamma_{5}\right\rangle
\end{aligned}
$$

we see that, again in virtue of the assumed finiteness of the Green's functions of the renormalized quark fields appearing in the r.h.s., the insertion of $k_{\mu} \int d^{4} x e^{-i k x} \hat{A}_{\mu}^{a}(x)$ is finite and, therefore, so is $\hat{A}_{\mu}^{a}(x)$.

From now on we will assume that the regularization has been performed so that assumption 1 is satisfied. We stress again that the recovery of chiral symmetry in the massless limit has nothing to do with it: if assumption t were not fulfilled, then all bare operators would be singular in the massless limit. As a consequence of assumption 4, the $a M_{0}$ dependence in $Z_{A}$ can be safely neglected, for asymptotically small $a$, and $Z_{A}$ will be a function of $g_{0}$ alone四.

\subsection{Ward Identities with Composite Operator Insertions}

When studying Ward Identities with composite operator insertions, another general property was needed in ref. [1], which we will now examine in full generality. It concerns the integrated insertion of $\bar{X}^{a}(y)$ together with a composite local operator.

\footnotetext{
${ }^{8}$ This behaviour is, of course, compatible with the possibility of spontaneous symmetry breaking.
} 
We will discuss the example of the regularized, integrated octet $(a \neq 0)$ axial Ward Identity [1]:

$$
\begin{aligned}
& -m_{q} Z_{p} \int d^{4} x\left\langle\bar{q}(x) \lambda^{a} \gamma_{5} q(x) \bar{q}(0) \frac{\lambda^{b}}{2} \gamma_{5} q(0) \Lambda_{n}(y)\right\rangle= \\
& =d^{a b c} Z_{p}\left\langle\bar{q}(0) \frac{\lambda^{c}}{2} q(0) \Lambda_{n}(y)\right\rangle+Z_{p}\left\langle\bar{q}(0) \frac{\lambda^{b}}{2} \gamma_{5} q(0) \delta_{A}^{a} \Lambda_{n}(y)\right\rangle+ \\
& +Z_{p} \int d^{4} x\left\langle\bar{X}^{a}(x) \bar{q}(0) \frac{\lambda^{b}}{2} \gamma_{5} q(0) \Lambda_{n}(y)\right\rangle
\end{aligned}
$$

where $Z_{p}\left(g_{0}, a \mu\right)$ is a logarithmically divergent renormalization constant which makes single insertions of the pseudoscalar density finite, $\Lambda_{n}(y)$ denotes a collection of $n q^{(r e n)}$ and $\bar{q}^{(r e n)}$ insertions, all at different points:

$$
\Lambda_{n}(y) \equiv q^{(r e n)}\left(y_{1}\right) \ldots \bar{q}^{(r e n)}\left(y_{n}\right)
$$

and $\delta_{A}^{a} \Lambda_{n}(y)$ its axial variation:

$$
\delta_{A}^{a} \Lambda_{n}(y) \equiv \gamma_{5} \frac{\lambda^{a}}{2} q^{(r e n)}\left(y_{1}\right) \ldots \bar{q}^{(r e n)}\left(y_{n}\right)+\ldots+q^{(r e n)}\left(y_{1}\right) \ldots \bar{q}^{(r e n)}\left(y_{n}\right) \gamma_{5} \frac{\lambda^{a}}{2}
$$

In order to study the $\bar{X}^{a}(x)$ insertion in the r.h.s of eq.(22) we start considering:

$$
\Phi^{a b} \equiv Z_{p} \int d^{4} y\left\langle\bar{O}_{5}^{a}(x) \bar{q}(0) \gamma_{5} \frac{\lambda^{b}}{2} q(0) \Lambda_{n}(y)\right\rangle
$$

Although $\bar{O}_{5}^{a}(x)$ and $Z_{p} \bar{q}(0) \gamma_{5} \frac{\lambda^{b}}{2} q(0)$, being renormalized operators, have finite insertions with fundamental fields, $\Phi^{a b}$ is still u.v. divergent due to short distance non-integrable singularities when $x \rightarrow 0$. We will consider here, for simplicity, the case $b \neq 0, a$. With an appropriate choice of $C_{s}$ we can construct out of $\Phi^{a b}$ a finite quantity as:

$$
\Phi_{R}^{a b}=\Phi^{a b}-Z_{p} Z_{5} \frac{C_{s}}{a} d^{a b c}\left\langle\bar{q}(0) \frac{\lambda^{c}}{2} q(0) \Lambda_{n}(y)\right\rangle
$$

As before we get a restriction on $C_{s}$ by applying $\left.\mu \frac{d}{d \mu}\right|_{g_{0}, M_{0}, a}$ to both sides of eq.(26):

$$
\begin{aligned}
& \left.\mu \frac{d}{d \mu}\right|_{g_{0}, M_{0}, a} \Phi_{R}^{a b} \equiv\left(\mu \frac{\partial}{\partial \mu}+\beta(g) \frac{\partial}{\partial g}\right) \Phi_{R}^{a b}= \\
& =-\left(\gamma_{\bar{O}_{5}}(g)+\gamma_{p}(g)+n \gamma_{q}(g)\right) \Phi_{R}^{a b}+ \\
& -\left.\frac{Z_{p} Z_{5}}{a} d^{a b c}\left\langle\bar{q}(0) \frac{\lambda^{c}}{2} q(0) \Lambda(y)\right\rangle \mu \frac{d}{d \mu}\right|_{g_{0}, M_{0}, a} C_{s}
\end{aligned}
$$

Eq.(27) shows that $C_{s}$ is a function of $g_{0}$ onlyf, so that:

$$
\begin{aligned}
& Z_{p} \int d^{4} x\left\langle\bar{X}^{a}(x) \bar{q}(0) \gamma_{5} \frac{\lambda^{b}}{2} q(0) \Lambda_{n}(y)\right\rangle= \\
& =\frac{a}{Z_{5}} \Phi^{a b}(x) \underset{a \approx 0}{\approx} Z_{p} C_{s}\left(g_{0}\right) d^{a b c}\left\langle\bar{q}(0) \frac{\lambda^{c}}{2} q(0) \Lambda_{n}(y)\right\rangle
\end{aligned}
$$

\footnotetext{
${ }^{9}$ The dependence on $a M_{0}$ can be neglected in view of assumption 4 .
} 
where we exploited the fact that $\frac{a}{Z_{5}} \Phi_{R}^{a b} \rightarrow 0$, as $a \rightarrow 0$

Eq.(28) fully confirms the results of ref.[1]. A similar analysis shows that, in general, for $a \neq 0$ and $b=0, \ldots, 8$, we have:

$$
\begin{aligned}
& -m_{q} \int d^{4} x\left\langle\bar{q}(x) \gamma_{5} \lambda^{a} q(x) P^{b}(0) \Lambda_{n}(y)\right\rangle= \\
& =d^{a b c}\left\langle S^{c}(0) \Lambda_{n}(y)\right\rangle+\left\langle P^{b}(0) \delta_{A}^{a} \Lambda_{n}(y)\right\rangle
\end{aligned}
$$

where $(a \neq 0)$ :

$$
\begin{aligned}
& P^{0}(x) \equiv Z_{p}\left(1+C_{p}\left(g_{0}\right)\right) \bar{q}(x) \gamma_{5} \frac{\lambda^{0}}{2} q(x) \\
& P^{a}(x) \equiv Z_{p} \bar{q}(x) \gamma_{5} \frac{\lambda^{a}}{2} q(x) \\
& S^{0}(x) \equiv Z_{p}\left[\left(1+C_{s^{0}}\left(g_{0}\right)\right) \bar{q}(x) \frac{\lambda^{0}}{2} q(x)+\frac{1}{3} \frac{D\left(g_{0}, a M_{0}\right)}{a^{3}}\right] \\
& S^{a}(x) \equiv Z_{p}\left(1+C_{s}\left(g_{0}\right)\right) \bar{q}(x) \frac{\lambda^{a}}{2} q(x)
\end{aligned}
$$

By evaluating eq.(29) in the chiral limit, $m_{q}=0$, we avoid an u.v. divergence in the l.h.s.ण, due to the simultaneous insertion of $\bar{q}(x) \gamma_{5} \lambda^{a} q(x)$ and $\bar{q}(0) \gamma_{5} \frac{\lambda^{b}}{2} q(0)$ and show that the $P$ 's and the $S$ 's, defined in eq.(30), belong to a renormalized $(3, \overline{3}) \oplus(\overline{3}, 3)$ representation of $S U(3) \otimes S U(3)$ [1].

Strictly speaking, the Ward Identity eq.(29) only provides a check that the $P^{a}(x)^{\prime}$ 's transform into the $S^{a}(x)$ 's (for $a=0, \ldots, 8$ ) under an axial transformation. In principle we should also consider the Ward Identity analogous to eq.(29), but with an $S^{b}(x)$ insertion in the 1.h.s. and check that in the r.h.s. the correct combination $d^{a b c} P^{c}(x)$ appears, without additional renormalization constants. This consistency is guaranteed by the fact that Ward identities are obtained by making a transformation, $\delta_{A}^{a} q(x)$ on the quark fields in the functional integral and the $\delta_{A}^{a}$ identically satisfy, on the lattice, the algebra of $S U(3) \otimes S U(3)$ :

$$
\begin{aligned}
& \delta_{A}^{a} \delta_{A}^{b}-\delta_{A}^{b} \delta_{A}^{a}=-i f^{a b c} \delta_{V}^{c} \\
& \delta_{V}^{a} \delta_{V}^{b}-\delta_{V}^{b} \delta_{V}^{a}=-i f^{a b c} \delta_{V}^{c}
\end{aligned}
$$

where $\delta_{V}^{a}$ denotes a vector flavor variation. It is easy to check that eq.(31) provides the required consistency. An explicit example of this kind of consistency, in a simpler case, will be given at the end of subsection 4.2.

\section{Global Anomalous Symmetries}

In this section we will discuss the definition and the renormalization of the Ward Identities related to the $U(1)$ chiral transformations [5].

\footnotetext{
${ }^{10}$ This divergence appears in the disconnected component of the Green' function and is relevant in the definition of the chiral condensate[1].
} 


\subsection{Anomalous Ward Identities with Elementary Opera- tor Insertions}

In analogy with the octet case, eq.(可), for a $U(1)$ chiral transformation we have the regularized identity:

$$
\begin{aligned}
& \partial_{x}^{\mu}\left\langle A_{\mu}(x) q(y) \bar{q}(z)\right\rangle=M_{0}\left\langle\bar{q}(x) \gamma_{5} q(x) q(y) \bar{q}(z)\right\rangle+ \\
& +\delta^{(4)}(x-y)\left\langle\frac{\gamma_{5}}{2} q(y) \bar{q}(z)\right\rangle+\delta^{(4)}(x-z)\left\langle q(y) \bar{q}(z) \frac{\gamma_{5}}{2}\right\rangle+ \\
& +\langle X(x) q(y) \bar{q}(z)\rangle
\end{aligned}
$$

where:

$$
A_{\mu}(x) \equiv \frac{1}{2} \bar{q}(x) \gamma_{\mu} \gamma_{5} q(x)
$$

As $X^{a}(x)$, also $X(x)$ has the form:

$$
X(x)=a O_{5}(x)
$$

In this case, however, in order to construct a finite operator out of $O_{5}(x)$, we have to perform more subtractions. Instead of eq.(10), we have:

$$
\begin{aligned}
& \bar{O}_{5}(x)=Z_{5}^{\prime}\left\{O_{5}(x)+\frac{\bar{M}^{\prime}}{a} \bar{q}(x) \gamma_{5} q(x)+\right. \\
& \left.+\frac{\left(Z_{A}^{\prime}-1\right)}{a} \partial^{\mu} A^{\mu}(x)-\frac{Z_{F \tilde{F}}}{a} F \tilde{F}\right\}
\end{aligned}
$$

where $F \tilde{F}$ is any formal regularization of the corresponding classical operator.

As in the octet case we conclude that $Z_{A}^{\prime}$ and $Z_{F \tilde{F}}$ are finite functions of $g_{0}$, while:

$$
\bar{M}^{\prime}=\frac{w^{\prime}\left(g_{0}, a M_{0}\right)}{a}
$$

Renormalizing the quark fields, eq.(33) becomes:

$$
\begin{aligned}
& Z_{A}^{\prime}\left(g_{0}\right) \partial_{x}^{\mu}\left\langle A_{\mu}(x) q^{(r e n)}(y) \bar{q}^{(r e n)}(z)\right\rangle= \\
& =m_{q}^{\prime}\left\langle\bar{q}(x) \gamma_{5} q(x) q^{(r e n)}(y) \bar{q}^{(r e n)}(z)\right\rangle+Z_{F}\left(g_{0}\right)\left\langle F \tilde{F}(x) q^{(r e n)}(y) \bar{q}^{(r e n)}(z)\right\rangle+ \\
& +\delta^{(4)}(x-y)\left\langle\frac{\gamma_{5}}{2} q^{(r e n)}(y) \bar{q}^{(r e n)}(z)\right\rangle+\delta^{(4)}(x-z)\left\langle q^{(r e n)}(y) \bar{q}^{(r e n)}(z) \frac{\gamma_{5}}{2}\right\rangle+ \\
& +\left\langle\bar{X}(x) q^{(r e n)}(y) \bar{q}^{(r e n)}(z)\right\rangle
\end{aligned}
$$

where:

$$
\begin{aligned}
& m_{q}^{\prime} \equiv M_{0}-\bar{M}^{\prime} \\
& \bar{X}(x) \equiv \frac{a}{Z_{5}^{\prime}} \bar{O}_{5}(x)
\end{aligned}
$$

Again, in eq.(38), the insertion of $\bar{X}(x)$ vanishes as $a \rightarrow 0$. An argument similar to the one used in the octet case shows the finiteness of the mass insertion[6]. However we cannot exclude separate logarithmically divergent contributions from $Z_{A}^{\prime}\left(g_{0}\right) \partial_{x}^{\mu} A_{\mu}(x)$ 
and $Z_{F \tilde{F}}\left(g_{0}\right) F \tilde{F}$. These divergences are, in fact, present [6] and require a logarithmically divergent mixing, in order to define separately finite operators:

$$
\begin{aligned}
& A_{\mu}^{R}(x) \equiv\left(1-Z_{C}\left(g_{0}, a \mu\right)\right) Z_{A}^{\prime}\left(g_{0}\right) A_{\mu}(x) \\
& F \tilde{F}^{R}(x) \equiv Z_{F \tilde{F}}\left(g_{0}\right) F \tilde{F}(x)-Z_{C}\left(g_{0}, a \mu\right) Z_{A}^{\prime}\left(g_{0}\right) \partial^{\mu} A_{\mu}(x)
\end{aligned}
$$

so that the renormalized anomalous Ward Identity with quark field insertions becomes:

$$
\begin{aligned}
& \partial_{x}^{\mu}\left\langle A_{\mu}^{R}(x) q^{(r e n)}(y) \bar{q}^{(r e n)}(z)\right\rangle= \\
& =m_{q}^{\prime}\left\langle\bar{q}(x) \gamma_{5} q(x) q^{(r e n)}(y) \bar{q}^{(r e n)}(z)\right\rangle+\left\langle F \tilde{F}^{R}(x) q^{(r e n)}(y) \bar{q}^{(r e n)}(z)\right\rangle+ \\
& +\delta^{(4)}(x-y)\left\langle\frac{\gamma_{5}}{2} q^{(r e n)}(y) \bar{q}^{(r e n)}(z)\right\rangle+\delta^{(4)}(x-z)\left\langle q^{(r e n)}(y) \bar{q}^{(r e n)}(z) \frac{\gamma_{5}}{2}\right\rangle
\end{aligned}
$$

In the anomalous case the normalization of the axial current is, of course, not fixed by Ward Identities, but must be adjusted according to some arbitrary prescription.

\subsection{Anomalous Ward Identities with Composite Operator Insertions}

In the presence of a composite operator, as, for instance, $P^{a}(y)$, defined in eq.(30), we can write the regularized and integrated $U(1)$ Ward Identity $\square$ :

$$
\begin{aligned}
& -\int d^{4} x\left\langle\left[m_{q}^{\prime} \bar{q}(x) \gamma_{5} q(x)+F \tilde{F}^{R}(x)\right] P^{a}(y) \Lambda_{n}(z)\right\rangle= \\
& =\left\langle P^{a}(y) \delta_{A} \Lambda_{n}(z)\right\rangle+Z_{p}\left\langle\bar{q}(y) \frac{\lambda^{a}}{2} q(y) \Lambda_{n}(z)\right\rangle \\
& +\int d^{4} x\left\langle\bar{X}(x) P^{a}(y) \Lambda_{n}(z)\right\rangle
\end{aligned}
$$

with an obvious meaning for $\delta_{A}$. For the insertion of $\bar{X}(x)$ in eq.42), we can construct an argument completely parallel to the one leading to eq.(28), which gives:

$$
\begin{aligned}
& \int d^{4} x\left\langle\bar{X}(x) P^{a}(y) \Lambda_{n}(z)\right\rangle \approx \\
& \underset{a \approx 0}{\approx} Z_{p} C^{\prime}\left(g_{0}\right)\left\langle\bar{q}(y) \frac{\lambda^{a}}{2} q(y) \Lambda_{n}(z)\right\rangle
\end{aligned}
$$

so that eq.(42) becomes:

$$
\begin{aligned}
& -\int d^{4} x\left\langle\left[m_{q}^{\prime} \bar{q}(x) \gamma_{5} q(x)+F \tilde{F}^{R}(x)\right] P^{a}(y) \Lambda_{n}(z)\right\rangle= \\
& =\left\langle P^{a}(y) \delta_{A} \Lambda_{n}(z)\right\rangle+Z_{p}\left(1+C^{\prime}\left(g_{0}\right)\right)\left\langle\bar{q}(y) \frac{\lambda^{a}}{2} q(y) \Lambda_{n}(z)\right\rangle \equiv \\
& \equiv\left\langle P^{a}(y) \delta_{A} \Lambda_{n}(z)\right\rangle+\frac{1+C^{\prime}\left(g_{0}\right)}{1+C_{s}\left(g_{0}\right)}\left\langle S^{a}(y) \Lambda_{n}(z)\right\rangle
\end{aligned}
$$

Through operator product expansion one can convince oneself that the double insertion of composite operators in eq.(44) is integrable without further subtractions. In

\footnotetext{
${ }^{11}$ We choose $a \neq 0$ in order to avoid disconnected contributions.
} 
fact in the product of $m_{q}^{\prime} \bar{q}(x) \gamma_{5} q(x)$ and $P^{a}(y)$ at $x \approx y$, the identity operator is missing because of the flavour structure $(a \neq 0)$ and the next contributing operator has dimension 3 , giving rise to an integrable singularity. A similar argument holds for the double insertion of $F \tilde{F}^{R}(x)$ and $P^{a}(y)$.

Eq.(44) is the prototype of the $U(1)$ integrated chiral Ward identities with composite operator insertions and can be used to solve the following consistency problem.

When sandwiched between on-shell states, $\hat{A}_{\mu}^{a}(x)$ and $A_{\mu}^{R}(x)$ satisfy the partial conservation equations:

$$
\begin{aligned}
& \partial^{\mu} \hat{A}_{\mu}^{a}(x)=m_{q} \bar{q}(x) \lambda^{a} \gamma_{5} q(x) \\
& \partial^{\mu} A_{\mu}^{R}(x)=m_{q}^{\prime} \bar{q}(x) \gamma_{5} q(x)+F \tilde{F}^{R}(x)
\end{aligned}
$$

While in eq.(46) the mass insertion is proportional to $m_{q}^{\prime} \equiv M_{0}-\bar{M}^{\prime}$, in eq.(45) it is proportional to $m_{q} \equiv M_{0}-\bar{M}$. Formally, in the continuum, the mass insertions in eqs. (45) and (46) have the same coefficient, the quark mass, so that they vanish together in the chiral limit.

We want to show that this is also true for the renormalized eqs.45) and (46): $m_{q}$ and $m_{q}^{\prime}$ are in fact proportional through a finite coefficient, so that both vanish in the chiral limit.

We integrate eq.(44) over $y$ :

$$
\begin{aligned}
& -\int d^{4} x d^{4} y\left\langle\left[m_{q}^{\prime} \bar{q}(x) \gamma_{5} q(x)+F \tilde{F}^{R}(x)\right] P^{a}(y) \Lambda_{n}(z)\right\rangle= \\
& =\int d^{4} y\left\langle P^{a}(y) \delta_{A} \Lambda_{n}(z)\right\rangle+Z_{p}\left(1+C^{\prime}\left(g_{0}\right)\right) \int d^{4} y\left\langle\bar{q}(y) \frac{\lambda^{a}}{2} q(y) \Lambda_{n}(z)\right\rangle
\end{aligned}
$$

and consider the chain of equalities:

$$
\begin{aligned}
& -\int d^{4} x d^{4} y\left\langle m_{q} \bar{q}(y) \lambda^{a} \gamma_{5} q(y)\left[m_{q}^{\prime} \bar{q}(x) \gamma_{5} q(x)+F \tilde{F}^{R}(x)\right] \Lambda_{n}(z)\right\rangle= \\
& =m_{q}^{\prime} \frac{1+C_{s}\left(g_{0}\right)}{1+C_{p}\left(g_{0}\right)} \int d^{4} x\left\langle\bar{q}(x) \lambda^{a} q(x) \Lambda_{n}(z)\right\rangle+ \\
& +\int d^{4} x\left\langle\left[m_{q}^{\prime} \bar{q}(x) \gamma_{5} q(x)+F \tilde{F}^{R}(x)\right] \delta_{A}^{a} \Lambda_{n}(z)\right\rangle= \\
& =m_{q}\left(1+C^{\prime}\left(g_{0}\right)\right) \int d^{4} y\left\langle\bar{q}(y) \lambda^{a} q(y) \Lambda_{n}(z)\right\rangle+ \\
& +\int d^{4} y\left\langle m_{q} \bar{q}(y) \lambda^{a} \gamma_{5} q(y) \delta_{A} \Lambda_{n}(z)\right\rangle
\end{aligned}
$$

Eq.(49) follows from eq.(48) through the octet Ward Identity, eq.(29), and the octet chiral invariance of $\int d^{4} x F F^{R}(x){ }^{\Gamma 2}$, while eq.(50) follows from eq.(48) through eq.(47).

The terms containing $\delta_{A}^{a} \Lambda_{n}(z)$ and $\delta_{A} \Lambda_{n}(z)$, in eqs. (49) and (50), can be further transformed. The integrated octet Ward Identity, eq.(20), in fact, gives:

$$
m_{q} \int d^{4} y\left\langle\bar{q}(y) \lambda^{a} \gamma_{5} q(y) \delta \Lambda_{n}(z)\right\rangle=-\left\langle\delta_{A}^{a} \delta_{A} \Lambda_{n}(z)\right\rangle
$$

while, integrating the $U(1)$ Ward Identity eq.(41), we get:

$$
\int d^{4} x\left\langle\left[m_{q}^{\prime} \bar{q}(x) \gamma_{5} q(x)+F \tilde{F}^{R}(x)\right] \delta^{a} \Lambda_{n}(z)\right\rangle=-\left\langle\delta_{A} \delta_{A}^{a} \Lambda_{n}(z)\right\rangle
$$

\footnotetext{
$12 \int d^{4} x F \tilde{F}^{R}(x)$ only depends on gluon fields.
} 
Since the octet and the $U(1)$ axial transformations commute:

$$
\delta_{A} \delta_{A}^{a}=\delta_{A}^{a} \delta_{A}
$$

eqs.(49) and (50) imply:

$$
m_{q}^{\prime} \frac{1+C_{s}\left(g_{0}\right)}{1+C_{p}\left(g_{0}\right)}=m_{q}\left(1+C^{\prime}\left(g_{0}\right)\right)
$$

which proves the required consistency.

We can further show that:

$$
C^{\prime}\left(g_{0}\right)=C_{s}\left(g_{0}\right)
$$

In fact eq. (44) tells us that ${ }^{\text {T3: }}$

$$
\begin{aligned}
& P^{a}(x) \\
& \frac{1+C^{\prime}\left(g_{0}\right)}{1+C_{s}\left(g_{0}\right)} S^{a}(x)
\end{aligned}
$$

belong to an irreducible, renormalized representation of the chiral $U(1)$ group. From the commutativity of $U(1)$ and octet transformations $(b \neq 0, a)$, eq.(53), we have:

$$
\begin{aligned}
& \delta \delta^{b} P^{a}(x)=\frac{1+C_{s}\left(g_{0}\right)}{1+C^{\prime}\left(g_{0}\right)} d^{b a c} P^{c}(x)= \\
& =\delta^{b} \delta P^{a}(x)=\frac{1+C^{\prime}\left(g_{0}\right)}{1+C_{s}\left(g_{0}\right)} d^{b a c} P^{c}(x)
\end{aligned}
$$

so that:

$$
\frac{1+C_{s}\left(g_{0}\right)}{1+C^{\prime}\left(g_{0}\right)}= \pm 1
$$

where the sign ambiguity can be reabsorbed into a redefinition of the abelian $U(1)$ charge, thus proving eq. (55).

\section{Acknowledgements}

I thank the CERN Theory Division for the kind hospitality.

I also thank Giancarlo Rossi for many discussions and suggestions and Laurent Lellouch for pointing out a mistake in an earlier version of the manuscript.

\footnotetext{
${ }^{13}$ We still consider $a \neq 0$, for simplicity.
} 


\section{References}

[1] M. Bochicchio, L. Maiani, G. Martinelli, G. C. Rossi, M. Testa, Nucl. Phys. B262 (1985) 331;

L. Maiani, G. Martinelli, G.C. Rossi, M. Testa, Phys. Lett. 176B (1986) 445;

L. Maiani, G. Martinelli, G.C. Rossi, M. Testa, Nucl. Phys. B289 (1987) 505

[2] G. Curci, Phys. Lett. B167 (1986) 425

[3] C. G. Callan, Phys. Rev. D2 (1970) 1541;

K. Symanzik, Comm. Math. Phys. 18 (1970) 227

[4] E. C. Poggio, H. R. Quinn, Phys. Rev. D14 (1976) 578

[5] For an early attempt see:

M. Bochicchio, G.C. Rossi, M. Testa, K. Yoshida, Phys. Lett. 149B (1984) 487

[6] W. A. Bardeen, Nucl. Phys. B75 (1974) 246 